\title{
RESEARCH
}

Open Access

\section{MiR-218 affects hypertrophic differentiation of human mesenchymal stromal cells during chondrogenesis via targeting RUNX2, MEF2C, and COL10A1}

Svitlana Melnik ${ }^{1 \dagger}$, Jessica Gabler ${ }^{1 \dagger}$, Simon I. Dreher ${ }^{1}$, Nicole Hecht ${ }^{1}$, Nina Hofmann ${ }^{1}$, Tobias Großner ${ }^{2}$ and Wiltrud Richter ${ }^{1 *}$

\begin{abstract}
Background: Human mesenchymal stromal cells (MSC) hold hopes for cartilage regenerative therapy due to their chondrogenic differentiation potential. However, undesirable occurrence of calcification after ectopic transplantation, known as hypertrophic degeneration, remains the major obstacle limiting application of MSC in cartilage tissue regeneration approaches. There is growing evidence that microRNAs (miRs) play essential roles in post-transcriptional regulation of hypertrophic differentiation during chondrogenesis. Aim of the study was to identify new miR candidates involved in repression of hypertrophy-related targets.

Methods: The miR expression profile in human articular chondrocytes (AC) was compared to that in hypertrophic chondrocytes derived from human MSC by microarray analysis, and miR expression was validated by qPCR. Putative targets were searched by in silico analysis and validated by miR reporter assay in HEK293T, by functional assays (western blotting and ALP-activity) in transiently transfected SaOS-2 cells, and by a miR pulldown assay in human MSC. The expression profile of miR-218 was assessed by qPCR during in vitro chondrogenesis of MSC and re-differentiation of AC. MSC were transfected with miR-218 mimic, and differentiation outcome was assessed over 28 days. MiR-218 expression was quantified in healthy and osteoarthritic cartilage of patients.

(Continued on next page)
\end{abstract}

\footnotetext{
* Correspondence: wiltrud.richter@med.uni-heidelberg.de

${ }^{\dagger}$ Svitlana Melnik and Jessica Gabler contributed equally to this work.

${ }^{1}$ Research Centre for Experimental Orthopaedics, Heidelberg University Hospital, Heidelberg, Germany

Full list of author information is available at the end of the article
}

(c) The Author(s). 2020 Open Access This article is licensed under a Creative Commons Attribution 4.0 International License, which permits use, sharing, adaptation, distribution and reproduction in any medium or format, as long as you give appropriate credit to the original author(s) and the source, provide a link to the Creative Commons licence, and indicate if changes were made. The images or other third party material in this article are included in the article's Creative Commons licence, unless indicated otherwise in a credit line to the material. If material is not included in the article's Creative Commons licence and your intended use is not permitted by statutory regulation or exceeds the permitted use, you will need to obtain permission directly from the copyright holder. To view a copy of this licence, visit http://creativecommons.org/licenses/by/4.0/. The Creative Commons Public Domain Dedication waiver (http://creativecommons.org/publicdomain/zero/1.0/) applies to the data made available in this article, unless otherwise stated in a credit line to the data. 
(Continued from previous page)

Results: Within the top 15 miRs differentially expressed between chondral AC versus endochondral MSC differentiation, miR-218 was selected as a candidate miR predicted to target hypertrophy-related genes. MiR218 was downregulated during chondrogenesis of MSC and showed a negative correlation to hypertrophic markers, such as COL1OA1 and MEF2C. It was confirmed in SaOS-2 cells that miR-218 directly targets hypertrophy-related COL1OA1, MEF2C, and RUNX2, as a gain of ectopic miR-218 mimic caused drop in MEF2C and RUNX2 protein accumulation, with attenuation of COL10A1 expression and significant concomitant reduction of ALP activity. A miR pulldown assay confirmed that miR-218 directly targets RUNX2, MEF2C in human MSC. Additionally, the gain of miR-218 in human MSC attenuated hypertrophic markers (MEF2C, RUNX2, COL1OA1, ALPL), although with no boost of chondrogenic markers (GAG deposition, COL2A1) due to activation of WNT/ $\beta$-catenin signaling. Moreover, no correlation between miR-218 expression and a pathologic phenotype in the cartilage of osteoarthritis (OA) patients was found.

Conclusions: Although miR-218 was shown to target pro-hypertrophic markers MEF2C, COL10A1, and RUNX2 in human MSC during chondrogenic differentiation, overall, it could not significantly reduce the hypertrophic phenotype or boost chondrogenesis. This could be explained by a concomitant activation of WNT/ $\beta$-catenin signaling counteracting the anti-hypertrophic effects of miR-218. Therefore, to achieve a full inhibition of the endochondral pathway, a whole class of anti-hypertrophic miRs, including miR-218, needs to be taken into consideration.

Keywords: miR-218, Mesenchymal stromal cells (MSC), Chondrocyte hypertrophy, Osteoarthritis (OA), MEF2C, RUNX2, Collagen type $X$

\section{Background}

Human bone marrow-derived mesenchymal stromal cells (MSC) are a promising cell source for tissue engineering of cartilage defects due to their multi-lineage differentiation capacity, including chondrogenic potential [1, 2]. However, in vitro MSC-generated chondrocytes by a classical TGF $\beta$-driven chondrogenesis protocol are characterized by unstable chondrocyte-like phenotype and undergo hypertrophic degeneration $[3,4]$. Hypertrophy is a terminal stage of chondrocyte differentiation and also is one of the prominent characteristics of osteoarthritis (OA) [5]. Expression of cartilage-specific collagen type II and proteoglycans in MSC-derived chondrocytes is accompanied by induction of hypertrophic markers, such as collagen type $\mathrm{X}$ and alkaline phosphatase (ALP) activity [3, 6], both associated with the differentiation cascade leading to mineralization and endochondral bone formation after ectopic transplantation. On the contrary, mature chondrocytes derived from the cartilage (defined as articular or hyaline chondrocytes, AC), cultured under similar conditions, remain negative for collagen type $\mathrm{X}$ and ALP and form stable non-calcified ectopic cartilage transplants [3].

The molecular mechanisms that are involved in the induction and regulation of chondrocyte hypertrophy are complex and remain poorly understood. Hypertrophic differentiation of chondrocytes is promoted or inhibited by multiple factors involved in different cell signaling pathways, including insulin-like growth factors (IGFs) [7], parathyroidhormone-related peptide (PTHrP) [8], Indian hedgehog $(\mathrm{IHH})$ [9], fibroblast growth factors (FGFs) [10], canonical and non-canonical WNT signaling [11], and transforming growth factor $\beta$ (TGF $\beta$ ) family members with bone morphogenetic proteins (BMPs) [12]. At a transcriptional level, Runt-related transcription factors 2 (RUNX2) and myocyte enhancer factor-2C (MEF2C) have been implicated as key transcription factors regulating chondrocyte hypertrophy, as they drive expression of the terminal differentiation markers, such as collagen type X [13-15], metalloproteinases MMP3 [16] and MMP13 [17-19], integrinbinding sialoprotein (IBSP) [20, 21], Indian hedgehog (IHH) [20, 22], and ALP [3, 20, 23, 24]. Studies in mouse models have demonstrated that several evolutionarily conserved Runx2 and Mef2c binding sites were found at the murine Col10a1 enhancer and promoter [15, 25], suggesting a direct regulatory role these transcription factors play in murine Col10a1 gene expression. Mef $2 c$ is expressed in pre-hypertrophic and hypertrophic chondrocytes, and its genetic deletion or expression of a dominant-negative $M e f 2 c$ mutant in endochondral cartilage impairs hypertrophy [13]. Additionally, Mef2c mutant mice showed a dramatically diminished expression of Runx2, suggesting that, epistatically, Runx2 acts downstream of Mef2c [13]. Remarkably, although it was believed that Runx2 and Runx3 play redundant roles in mouse chondrocyte maturation $[9,26]$, in human MSC, RUNX3, but not RUNX2, has been demonstrated to be the key transcription factor, together with $\mathrm{MEF} 2 \mathrm{C}$, driving hypertrophy and regulating the endochondral pathway in MSC, whereas RUNX2 has been rather assigned as an antichondrogenic factor [20]. 
Numerous studies (reviewed in [27]) suggest that microRNAs (miRs) play an important role in chondrocyte biology by suppressing their target gene expression at the post-transcriptional level. MicroRNAs are 22 nucleotidelong noncoding RNAs that directly bind to target mRNAs in a sequence-complimentary manner to facilitate the degradation of their target transcripts. To date, there is limited knowledge of miRs that are directly involved in posttranscriptional regulation of hypertrophy-related mRNAs during chondrogenesis. It has been suggested that a number of different miRs might target Runx2 in the murine chondrogenic cell line ATDC5 [28]. Of those, miR-218 (other name: hsa-miR-218) was demonstrated to directly target RUNX2 in human osteogenic dental stem cells (DSC) [29] and HPGD (15-hydroxyprostaglandin dehydrogenase) in rabbit synovium-derived MSC promoting their chondrogenic differentiation [30]. Additionally, miR-29b in murine MSC has been demonstrated to promote the hypertrophic phenotype [31], and miR-381 resembled anti-hypertrophic properties in the human chondrosarcoma cell line SW1353 [32].

In our previous study, we have identified a set of differentially expressed miRs during in vitro chondrogenesis of human MSC and defined miR clusters specific for different stages of chondrogenic differentiation (prechondrocytes, chondroblasts, chondrocytes, and hypertrophic chondrocytes) [33]. However, it was not clear whether among the identified miR clusters were those related to hypertrophy onset. Also, no mRNA targets were characterized by those differentially expressed miRs.

In the present study, we addressed the question which miRs are directly involved in post-transcriptional regulation of hypertrophy-related genes. For this, human bone marrow-derived MSC were subjected to TGF $\beta$-driven chondrogenesis resulting in hypertrophic chondrocytes. Their miR expression profile was determined by microarray analysis and compared to that of re-differentiated non-hypertrophic human articular chondrocytes (AC). We identified miR-218 as the best candidate to target hypertrophic genes and validated its function during human MSC chondrogenesis. This allowed us to draw conclusions about a role of this $\mathrm{miR}$ in the hypertrophic phenotype regulation during chondrogenic differentiation of human MSC.

\section{Material and methods}

\section{Cell lines, isolation of MSC and AC, and cell expansion}

Human cell lines: embryonic kidney epithelial HEK293T and bone osteosarcoma SaOS-2 were from the American Type Culture Collection (ATCC), and cells were grown in DMEM (Gibco) supplied with $10 \%$ fetal calf serum (FCS, Sigma), 100 I.U./mL penicillin, $100 \mu \mathrm{g} / \mathrm{mL}$ streptomycin (Biochrome).
MSC were isolated from fresh bone marrow aspirates of patients undergoing elective bone surgery $(N=17$ donors). AC were isolated from knee cartilage of tibia plateau that was removed due to OA ( $N=13$ donors), or from healthy donors $(N=21)$, either undergoing arthroscopic surgery of a knee due to trauma, or from deceased due to unrelated causes patients. The healthy articular cartilage was carefully removed from regions with no macroscopically evident degeneration and washed with phosphate-buffered saline (PBS), to avoid contamination by other cells.

The study was approved by the local ethics committee (Medical Faculty of the University of Heidelberg), and the informed consent was obtained from all the patients participated in the study, according to the 1964 Declaration of Helsinki, updated in 2000. All cells used for experiments in the study were from HIV-, HBV-, and $\mathrm{HCV}$-negative donors.

MSC population was isolated, as described before [12]. In brief, the mononuclear cell fraction was separated from bone marrow aspirates by Ficoll-Paque $\mathrm{e}^{\mathrm{mm}}$ densitygradient and seeded at density of $1.25 \times 10^{5}$ cells $/ \mathrm{cm}^{2}$ into $0.1 \%$ gelatin-coated culture flasks in expansion medium (DMEM high glucose, 12.5\% FCS, 100 I.U./mL penicillin, $100 \mu \mathrm{g} / \mathrm{mL}$ streptomycin, $2 \mathrm{mML}$-glutamine, $1 \%$ non-essential amino acids, $0.1 \% \beta$-mercaptoethanol (all from Life Technologies), and $4 \mathrm{ng} / \mathrm{mL}$ FGF-2 (Active Bioscience, Germany)). After $24 \mathrm{~h}$, non-adherent cells were removed by washing with PBS. The medium was replaced 3 times a week, and cells were expanded until passage 3 . For passaging, once cells reached $80 \%$ of confluence, they were detached with trypsin/EDTA and plated at density $5000 \mathrm{cells} / \mathrm{cm}^{2}$.

For articular chondrocyte isolation, cartilage pieces were minced, and chondrocytes were released by overnight digestion with collagenase B (Roche, Germany) and hyaluronidase (Sigma-Aldrich, Germany), as described before [34]. Chondrocytes were expanded in DMEM medium containing 10\% FCS, $100 \mathrm{IU} / \mathrm{mL}$ penicillin, $100 \mu \mathrm{g} / \mathrm{mL}$ streptomycin, at $37^{\circ} \mathrm{C}, 6 \% \mathrm{CO}_{2}$. The medium was changed twice per week, and cells were expanded until passage 2 .

\section{Chondrogenic differentiation (MSC) and re-differentiation} (AC)

For chondrogenic differentiation (MSC) or re-differentiation (AC), at the 3rd (MSC) or 2nd (AC) passages, $5 \times 10^{5}$ of MSC or AC were resuspended in chondrogenic medium (DMEM high glucose, $0.1 \mu \mathrm{M}$ dexamethasone, $0.17 \mathrm{mM}$ ascorbic-acid 2-phosphate, $5 \mathrm{mg} / \mathrm{ml}$ transferrin, $5 \mathrm{ng} / \mathrm{ml} \mathrm{so-}$ dium selenite, $1 \mathrm{mM}$ sodium pyruvate, $0.35 \mathrm{mM}$ proline, $1.25 \mathrm{mg} / \mathrm{ml}$ bovine serum albumin (BSA), 100 I.U./mL penicillin, $100 \mu \mathrm{g} / \mathrm{mL}$ streptomycin, $5 \mathrm{mg} / \mathrm{ml}$ insulin (SanofiAventis, Germany), and $10 \mathrm{ng} / \mathrm{ml}$ TGF- $\beta 1$ (PeproTech, 
Germany)). Cells were centrifuged at $500 \mathrm{~g}$ for $5 \mathrm{~min}$, or allowed to self-aggregate without centrifugation, to generate high-density pellets. Pellets were cultured for 3, 4, or 6 weeks at $37^{\circ} \mathrm{C}, 6 \% \mathrm{CO}^{2}$ with medium change 3 times a week.

\section{MicroRNA microarray analysis}

Five pellets per donor (MCS, day 42, $N=3$; AC, day 21, $N=1$ ) were pooled, mechanically minced in a polytron, and total RNA was isolated by guanidinium thiocyanate/ phenol extraction with peqGOLD Trifast (PeqLab, Germany). Isolated total RNA was used for miR expression analysis with an Agilent Human microRNA Microarray v16.0 (1349 miRs; Agilent Technologies, USA). The quality control of total RNA, labeling, array hybridization, and microarray scanning was performed at the DKFZ Genomics Core Facility (Heidelberg, Germany). Microarray scanning was done using an iScan array scanner. Data extraction was done for all beads individually, and outliers were removed when the absolute difference to the median was greater than 2.5 times MAD (mean of absolute deviation) (2.5 Hampel's method). All remaining bead level data points were then quantile normalized. As a test for significance, the Student's $t$ test was used on the bead expression values of the two groups of interest, and Benjamini-Hochberg correction [35] was applied to the complete set of $p$ values of all ProbeIDs on the chip. The average expression value was calculated as the mean of the measured expressions of beads, together with the standard deviation of the beads.

\section{In silico target prediction analysis and KEGG pathway analysis}

MicroRNA target prediction tools TargetScan, MiRWalk, and miRanda were applied to identify putative mRNA targets for miR-218. Putative mRNA targets with strong mirSVR (microRNA score vector regression) downregulation score (- 0.4, http://www.microRNA.org) suggested by all used prediction tools were included in further consideration and analysis. For systematic analysis of GO (gene ontology) terms and KEGG (Kyoto Encyclopedia of Genes and Genomes) signaling pathways of putative mRNA targets suggested by the miR prediction tools, the DAVID (Database for Annotation, Visualization, and Integrated Discovery) gene functional classification tool was applied, with a stringency set at the highest level [36].

\section{RNA extraction and quantitative reverse-transcriptase PCR (qRT-PCR)}

Total RNA was isolated from cells and pellets using a standard guanidiniumthiocyanate/phenol extraction protocol with peqGOLD TriFast ${ }^{\mathrm{Ts}}$ reagent (Peqlab, Erlangen, Germany). Total RNA from cartilage tissue was isolated, as described before [37, 38]. Polyadenylated mRNA was isolated using oligo $\mathrm{d}(\mathrm{T})$-coupled magnetic beads (Dynabeads, Dynal, Invitrogen $\mathrm{GmbH}$, Karlsruhe, Germany), according to the manufacturer's instruction. For the first-strand cDNA synthesis, $20 \mathrm{ng}$ of mRNA was used with reverse transcriptase $\left(\right.$ Omniscript $^{\oplus}$, Qiagen, Hilden, Germany) and oligo-d(T) primers. Quantitative reversed transcriptase PCR (qRT-PCR) was performed using the SYBR green I mix (ThermoScientific, Rockford, USA) and gene-specific primers (Table S1) with StratageneMx3000P (Agilent Technologies, Böblingen, Germany).

For the quantification of microRNAs expression, $10 \mathrm{ng}$ of total RNA was used for cDNA synthesis with the TaqMan ${ }^{\oplus}$ MicroRNA Reverse Transcription Kit (ThermoFisher Scientific). Relative expression levels were determined by qRT-PCR using the miR detection method from the Applied Biosystems (MicroRNA Reverse Transcription Kit) combined with the TaqMan MicroRNA Assays, using primers for U6 (Assay ID: 0001973) and hsa-miR-218-5p (Assay ID: 000521). Expression levels of mRNA and miR were calculated using 2- $\triangle \triangle \mathrm{CT}$ method [39] and normalized to housekeeping genes Actin Beta $(A C T B)$, Hypoxanthine-guanine phosphoribosyltransferase (HPRT), Ribosomal Protein L13 (RPL13), and Cleavage and Polyadenylation Specific Factor 6 (CPSF6) (for mRNAs), or U6 (Small Nuclear Ribonucleoprotein U6, SNRNU6) (for miRs).

\section{3'UTR luciferase reporter assay}

The 3'UTR of COL1OA1 and MEF2C containing a seed region for miR-218 were amplified from genomic DNA by PCR. The 3'UTR fragment was cloned into SpeI and HindIII restriction sites of pMIR-REPORT Luciferase vector (ThermoFisher Scientific). The 3'UTR of RUNX2 containing a seed region for miR-218, as well as all mutated sequences, were chemically synthesized (GeneArt, Germany). Primers used for this assay are listed in Table S2.

For miR gain-of-function experiments, microRNA mimics and the miR-218 inhibitor (anti-miR-218) were purchased from Ambion (mirVana: MC10328, MH10328, 4464058).

HEK293T cells, seeded a day before transfection at a density $5 \times 10^{4}$ cells/well, were cultured on 24 well plates. Co-transfection of $50 \mathrm{nM}$ of a selected miR mimic combined with $250 \mathrm{ng}$ of a corresponding tested reporter construct, together with $250 \mathrm{ng}$ of a normalization control $\beta$-Gal vector (ThermoFisher Scientific), was carried out using Lipofectamine 2000 reagent (Invitrogen, Germany). Luciferase activity was measured $48 \mathrm{~h}$ after transfection with Victor3 Multilabel Counter 1420-042 using the Luciferase Reporter Assay Kit (Promega, USA). Luciferase intensity signals were normalized to the $\beta$ Galactosidase activity in the same sample. Three 
independent experiments with six biological replicates were performed for this assay.

\section{Ectopic overexpression of miR-218 in SaOS-2 and MSC cells}

For miR overexpression in SaOS-2 and MSC cells, electroporation (in case of SaOS-2) (Neon, ThermoFisher Scientific), or transfection with Dharmafect1 (Dharmacon) (in case of SaOS-2 or MSC cells) were carried out, according to the manufacturers' instruction. In brief, for electroporation, $1 \times 10^{6}$ cells mixed with $10 \mu \mathrm{M}$ of miR mimic in $100 \mu \mathrm{l} \mathrm{R}$-buffer were electroporated with two pulses at $1200 \mathrm{~V}$ for $15 \mathrm{~ms}$. For a reverse method of transfection using Dharmafect $1,10^{6}$ cells (SaOS-2 or MSC) resuspended in $100 \mu$ l of DMEM medium containing no additives, were transfected with $50 \mathrm{nM}$ of miR mimic, according to the manufacturer's protocol. In case of MSC, transfected cells were transferred in chondrogenic medium and subjected to chondrogenic differentiation for 28 days, as described above. SaOS-2 cells were harvested $72 \mathrm{~h}$ after transfection for Western blotting and qRT-PCR analyses.

\section{MiR pulldown assay}

For the miR pulldown assay, MSC at passage 3 were harvested, washed 2 timed in ice-cold PBS and lysed (100 $\mu \mathrm{l}$ of buffer per $10^{6}$ cells) in RNase-free lysis buffer containing $10 \mathrm{mM}$ HEPES (pH 7.9), $10 \mathrm{mM} \mathrm{KCl,} 1.5 \mathrm{mM} \mathrm{MgCl}_{2}$, $10 \mathrm{mM}$ DTT, $10 \mathrm{mM}$ PMSF, 1/100 of Halt ${ }^{\mathrm{TM}}$ protease and phosphatase inhibitor-cocktails (PIC) (ThermoFisher Scientific), 1/100 of RNaseOUT (Invitrogen) for $30 \mathrm{~min}$ on ice. The cell lysate was cleared by centrifugation at $5000 \mathrm{~g}$ for $5 \mathrm{~min}$, and the concentrations of $\mathrm{KCl}$ and $\mathrm{MgCl}_{2}$ were adjusted to $100 \mathrm{mM}$ and $5 \mathrm{mM}$, correspondingly. NP40 was added to $0.5 \%$, and the cell lysate was aliquoted, with preservation of an aliquot for the input assessment. To each aliquot, $1 \mu \mathrm{M}$ of either miR-218 or a control non-targeting (NC) biotin-labeled miR mimic was added (biotin hsa-miR-218-5p miRCURY LNA Premium miRNA Mimic: YM00471984-BDI, and Negative Control miRCURY LNA miRNA Mimic: YM00479902BDI, Qiagen) and incubated for $4 \mathrm{~h}$ at $4{ }^{\circ} \mathrm{C}$. Next, $20 \mu \mathrm{l}$ of pre-blocked (for $1 \mathrm{~h}$ in lysis buffer containing $250 \mu \mathrm{g}$ RNase-free BSA (NEB) and $100 \mu \mathrm{g}$ yeast tRNA (ThermoFisher Scientific)) streptavidin magnetic beads (Roche) were added to the cell lysate aliquots and incubated overnight. Then, beads were washed 5 times in RNasefree IP buffer (50 mM Tris- $\mathrm{HCl}$ (pH 7.4), $100 \mathrm{mM} \mathrm{KCl}$, $5 \mathrm{mM} \mathrm{MgCl}_{2}, 0.5 \% \mathrm{NP} 40,10 \mathrm{mM}$ DTT, $10 \mathrm{mM}$ PMSF). Total RNA was extracted from beads using peqGOLD TriFast $^{\mathrm{TM}}$ reagent (Peqlab, Erlangen, Germany) and processed for RNA analysis by quantitative reverse transcriptase polymerase chain reaction (qRT-PCR), as described above, omitting the isolation of polyadenylated mRNA using oligo $\mathrm{d}(\mathrm{T})$-coupled magnetic beads step.

\section{Western blotting (WB)}

Cells and pellets were lysed in lysis buffer containing 50 $\mathrm{mM}$ Tris- $\mathrm{HCl}$ (pH 7.4), $150 \mathrm{mM} \mathrm{NaCl}, 1 \%$ Triton X-100, for $5 \mathrm{~min}$ on ice, with a preliminary grinding step (in case of pellets) using Powerful ball mill Retsch MM400 ( 2 rounds for $2 \mathrm{~min}, 30 \mathrm{~Hz}$ ). For analyses of cytosolic $\beta$ catenin, cell extracts were prepared in Saponin lysis buffer $(0.05 \%$ saponin, $1 \mathrm{mM} \mathrm{MgCl} 2,50 \mathrm{mM}$ Tris- $\mathrm{HCl}(\mathrm{pH}$ 7.4), $150 \mathrm{mM} \mathrm{NaCl}, 2.75 \mathrm{mM} \quad \mathrm{KCl}, \quad 0.1 \mathrm{mM} \quad \beta-$ mercaptoethanol) [40]. Cell lysates were cleared by centrifugation, and proteins were resolved by SDS-PAGE, blotted onto a nitrocellulose membrane and analyzed by WB. Following antibodies were used: MEF2C (Cell Signaling, D80C1), RUNX2 (MBL, D130-3), $\beta$-catenin (BD Transduction Laboratories, 610153), $\alpha$-tubulin (ThermoFisher Scientific, MS-581-P0), and $\beta$-actin (AC-15, GeneTex, USA).

\section{ALP activity assay}

ALP activity was measured by colorimetric assay using multi-mode plate reader FLUOstar Omega (BMG Labtech). For this, cell culture supernatants (5 per group or treatment), conditioned for 2 days, were collected and mixed with an equal volume $(100 \mu \mathrm{l})$ of ALP substrate solution $(10 \mathrm{mg} / \mathrm{ml}$ p-nitrophenylphosphate in $0.1 \mathrm{M}$ glycine, $1 \mathrm{mM} \mathrm{MgCl}_{2}$, and $1 \mathrm{mM} \mathrm{ZnCl} 2, \mathrm{pH}$ 9.6). After incubation for $180 \mathrm{~min}$, absorbance recorded at $405 \mathrm{~nm}$ was corrected for a signal at $490 \mathrm{~nm}$, and enzyme activity was quantified using a standard curve built using serial dilutions of p-nitrophenol (Sigma Aldrich). Six independent experiments with two technical replicates were performed for this assay.

\section{Histology and immunohistochemistry}

Pellets were fixed for $24 \mathrm{~h}$ in $4 \%$ formaldehyde, dehydrated in a graded isopropanol series, and paraffinembedded. Thin sections $(5 \mu \mathrm{m})$ were stained with Safranin $\mathrm{O}\left(0.2 \%\right.$ in $1 \%$ acetic acid) using Certistain ${ }^{\bullet}$ fast green $(0.04 \%$ in $0.2 \%$ acetic acid) for counter-staining, according to a standard procedure. Immunohistochemical detection of collagens, type II and type $\mathrm{X}$, was performed as described [41]. Briefly, sections treated with $4 \mathrm{mg} / \mathrm{mL}$ hyaluronidase in PBS, $\mathrm{pH} 5.5$, and $1 \mathrm{mg} /$ $\mathrm{mL}$ pronase, then blocked with $5 \%$ BSA and stained with collagen type II antibody (clone II-4C11, ICN Biomedicals), or collagen type X antibody (clone X53, Quartett, Germany), followed by incubation with biotinylated goat anti-mouse antibody (1:500, Dianova), and subsequent detection with streptavidin alkaline-phosphatase fast red (ThermoFisher Scientific). 


\section{Quantification of proteoglycan content (DMMB assay)}

Proteoglycan content in the cartilaginous tissue was measured by DMMB (dimethyl-methylene blue) assay, as described before [42]. For this, pellets $(N=2$ per donor) were harvested at day 28 of the chondrogenic induction, washed with PBS, and digested overnight in $1 \mathrm{ml}$ of lysis buffer containing $50 \mathrm{mM}$ Tris- $\mathrm{HCl}(\mathrm{pH} 8.0)$, and $1 \mathrm{mM} \mathrm{CaCl}_{2}$ with $500 \mu \mathrm{g} / \mathrm{ml}$ Proteinase $\mathrm{K}$ (Roche, Mannheim, Germany) at $60^{\circ} \mathrm{C}$. Pellets digests $(30 \mu \mathrm{l})$ were mixed with $200 \mu \mathrm{l}$ of DMMB solution $(38 \mu \mathrm{M}$ dimethyl-methylene blue, Sigma, $40 \mathrm{mM}$ glycine, $40 \mathrm{mM}$ $\mathrm{NaCl}$ ). Proteoglycan content was measured by spectrophotometry at $540 \mathrm{~nm}$ and quantified using a standard curve built with chondroitin sulphate substrate standard. The values were normalized to DNA amount in lysed cells measured using Quanti-iT PicoGreen dsDNA kit (Invitrogen, Eugene, USA). For this, $20 \mu \mathrm{l}$ of the digested pellet sample were mixed with $80 \mu \mathrm{TE}$ buffer $(200 \mathrm{mM}$ Tris- $\mathrm{HCl}$ (pH 7.4), 20 mM EDTA) and PicoGreen solution, and fluorescence in samples was measured at 485/ $535 \mathrm{~nm}$.

\section{Statistical analysis}

Statistical analysis was performed using GraphPad (Prism) software, with application of either MannWhitney $U$ test, or two-tailed Student's $t$ test; expression kinetic curves were tested with paired two-tailed Student's $t$ test; $p$ values $\leq 0.05$ were referred to as being significant.

\section{Results}

Identification and selection of differentially expressed miRs between hypertrophic and articular chondrocytes

To identify miRNAs that affect hypertrophy-associated signaling pathways, we used a direct comparison of miR expression profiles between hypertrophic and articular chondrocytes by microarray analysis. Upon chondrogenic in vitro differentiation in $3 \mathrm{D}$ culture, human bone marrow MSC undergo hypertrophy, whereas AC always remain negative for hypertrophic markers after redifferentiation at the same conditions. Indeed, when the human bone marrow MSC that underwent chondrogenic differentiation were compared to re-differentiated $\mathrm{AC}$ isolated from the knee cartilage, these two cell populations produced cartilaginous tissue cultures in pellets of distinct phenotypes (Fig. 1). Both resulting tissues showed pronounced staining with Safranin O, indicating a successful progression of chondrogenesis and proteoglycan deposition in both groups. This result was additionally supported by comparable levels of collagen type II immunostaining (Fig. 1a). However, in contrary to this, immunostaining for the hypertrophic marker collagen type $\mathrm{X}$ was positive in MSC and negative in $\mathrm{AC}$, indicating that MSC, but not $\mathrm{AC}$, underwent hypertrophic differentiation. This conclusion was also supported by mRNA expression data for COL10A1 (Fig. 1b). Expression levels of other hypertrophy-associated markers, such as $M E F 2 C, I H H$, and $R U N X 2$, were also significantly increased in MSC-derived chondrocytes in comparison to AC (Fig. 1b), and this was accompanied by significantly higher ALP activity in culture supernatants of pellet cultures formed by MSC in relation to a low enzyme activity for AC pellets (Fig. 1c). Collectively, these data highlight that chondrocytes derived from MSC or $\mathrm{AC}$ have different phenotypes in regard of hypertrophy, and these cells could serve as different chondrocyte cell populations to address whether specifically regulated microRNAs are related to induction of the hypertrophic phenotype.

Next, RNA samples isolated from pellets of chondrogenically differentiated MSC or AC were analyzed by a microRNA microarray. Among 1349 miR probes tested, 115 miRs showed more than 2-fold differences in their expression levels between MSC versus AC, with the top 15 reaching over 60 -fold changes between MSC and AC (Table 1). These top 15 differentially expressed miRs were then subjected to in silico analysis with application of a single criterion: their putative mRNA targets had to be involved in regulation of hypertrophic pathways. Three microRNA target prediction tools: TargetScan, MiRWalk, and miRanda were utilized to identify putative mRNA targets. Among these 15 candidates, miR-218 was predicted to potentially target the bona fide hypertrophy markers MEF2C, RUNX2, and COL1OA1 with a substantial miRSVR (microRNA score of vector regression). MirSVR computed by http://www.microRNA.org tool uses vector regression (SVR) to evaluate a wide range of features allowing prediction and ranking of multiple putative microRNA target sites' efficiencies. It serves as a measure of the likelihood that a given miR interacts with a certain sequence of mRNA target. It takes into account a secondary structure accessibility of the site, its conservation that affect miR-mRNA pairing, and a score lower than -0.4 is generally regarded as a significant one (Table 2).

\section{Reversed correlation between miR-218 and hypertrophic marker expression kinetic profiles during MSC chondrogenesis}

Next, we validated the microarray results using qRTPCR and also monitored the expression of miR-218 in MSC-derived chondrocytes at different stages of chondrogenesis and of re-differentiation of $\mathrm{AC}$ from four to six different donors. MiR-218 was already more expressed in expanded MSC in comparison to AC. Along the time-course of chondrogenesis, expression of miR-218 gradually declined: high expression levels at a start of chondrogenesis (day 0) later were reduced at 
a
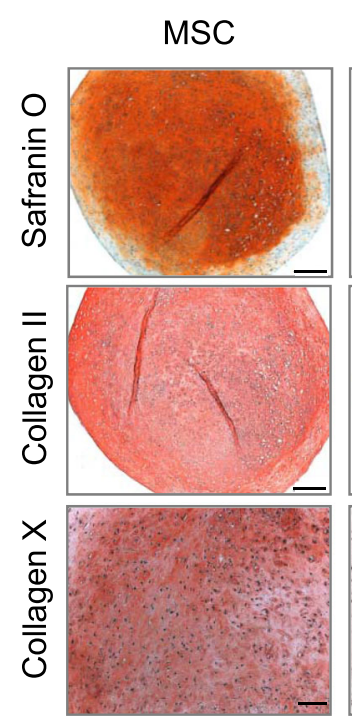

b

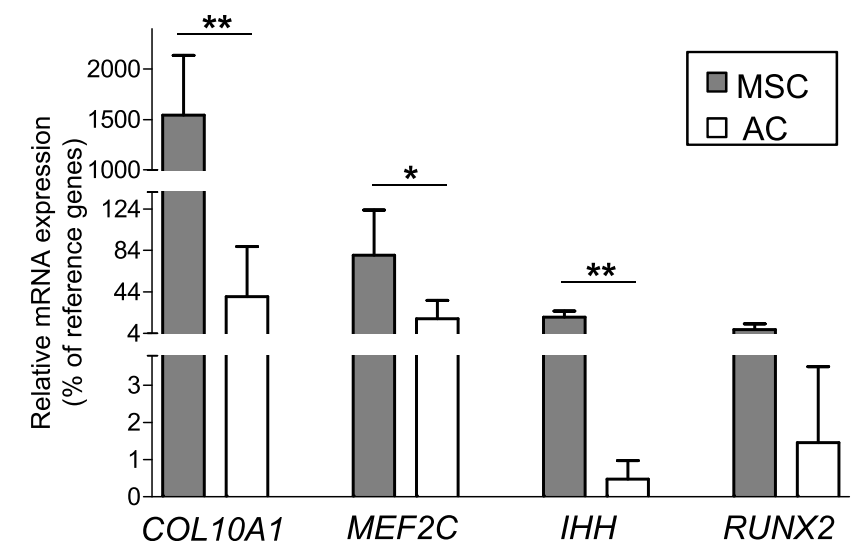

Fig. 1 MSC, but not AC, undergo hypertrophy under chondrogenic conditions. MSC ( $N=6$ donors) or $A C$ ( $N=4$ donors) were subjected to chondrogenic conditions and evaluated at the end of their chondrogenesis. a Representative images for Safranin $\mathrm{O}$ staining, and collagen type $\|$ or type X immunohistochemistry. Scale bar, $100 \mu \mathrm{m}$. b mRNA expression for indicated genes was measured by qRT-PCR, and values were normalized to three housekeeping genes (ACTB, HPRT, RLP13). c Alkaline phosphatase activity (ALP) measured in culture media from cell pellets. Mean values \pm SD are shown (MSC-gray, AC—white); ${ }^{*} p \leq 0.05,{ }^{* *} p \leq 0.01$ (Mann-Whitney $U$ )

days 21 and 42 (Fig. 2a), but always remained significantly above AC levels. Remarkably, the expression kinetic profiles for miR-218 and hypertrophic markers $M E F 2 C$ and $C O L 10 A 1$ showed a reverse regulation over 42 days of chondrogenesis (Fig. 2b). Additionally, expression of COL1OA1 and miR-218 showed a tight negative correlation (Pearson's correlation coefficient $r=-0.5664$ with $p=0.0092$ ) during MSC chondrogenesis (Fig. 2c). These results imply a putative regulatory role of miR218 in post-transcriptional regulation of $M E F 2 C$ and COL10A1 genes and its anti-hypertrophic function.

\section{MiR-218 directly targets hypertrophic marker genes}

To understand the regulatory role of miR-218, we searched for additional mRNA targets, beyond of $M E F 2 C$, RUNX2, and COL1OA1, using different web- based bioinformatics tools: TargetScan, MiRWalk, and miRanda. This resulted in 4434 high-confidence hits for miR-218, matched by all three algorithms. Gene Ontology (GO) and KEGG (Kyoto Encyclopedia of Genes and Genomes) pathway analyses revealed that among the functional and signaling pathways regulated by these putative targets, there was a significant enrichment for genes related to "signal transduction" (including known hypertrophy-regulating signaling pathways, e.g., WNT, TGF $\beta$, HH (Hedgehog), and Thyroid hormone), "Signaling molecules and interaction" (including extracellular matrix (ECM) organization) and "Glycan biosynthesis and metabolism" (including glycosaminoglycan biosynthesis - chondroitin sulfate/dermatan sulfate) categories (Table 3). Altogether, this suggested a high complexity of putative miR-218 regulation mechanisms. 
Table 1 Top 15 differentially expressed microRNAs between redifferentiated articular chondrocytes (AC) and hypertrophic chondrocytes produced from MSC, sorted according to their regulation factor

\begin{tabular}{llll}
\hline miR & AC & MSC & Factor \\
\hline hsa-miR-181b & 0.1 & 280 & 2802 \\
hsa-miR-181a & 0.1 & 144 & 1443 \\
hsa-miR-513a-5p & 0.1 & 37 & 375 \\
hsa-miR-224 & 0.1 & 33 & 334 \\
hsa-miR-1208 & 0.1 & 23 & 227 \\
hsa-miR-4327 & 0.1 & 22 & 218 \\
hsa-miR-3188 & 0.1 & 19 & 187 \\
hsa-miR-218 & $\mathbf{0 . 1}$ & $\mathbf{1 9}$ & $\mathbf{1 8 5}$ \\
hsa-miR-145 & 0.1 & 16 & 156 \\
hsa-miR-181a & 20 & 2898 & 146 \\
hsa-miR-34b & 0.1 & 13 & 126 \\
hsa-miR-449c & 0.1 & 12 & 121 \\
hsa-miR-1226 & 0.1 & 7 & 73 \\
hsa-miR-449b & 0.1 & 6 & 62 \\
hsa-miR-4254 & 0.1 & 6 & 60 \\
\hline
\end{tabular}

Since miR-218 directly targeted the transcription factor RUNX2 in a number of mouse cells lines: osteoblasts (MC3T3-E1), chondrocytes (ATDC5), fibroblasts (NIH 3T3) [28], and human dental stem cells [29], we used it as a positive control mRNA target. A fragment of the RUNX2 3'UTR containing the predicted miRNA-218 seed region was cloned downstream of a CMV-driven firefly luciferase vector, and luciferase activity was measured after co-transfection with miR-218 mimic in HEK293T cells. To confirm the exact miR-218 binding region for $R U N X 2$, a reporter vector with a mutated seed-region in the 3'UTR was used as a negative binding control. A non-targeting (NC) miR mimic was used as another negative control. Indeed, ectopic miR-218 mimic could significantly reduce activity of the tested RUNX2 3'UTR reporter (Fig. 3a) demonstrating that miR-218 directly regulates expression of $R U N X 2$, as expected. This effect was abrogated by mutations introduced into the seed region for miR-218 in the 3'UTR of RUNX2. Also, no inhibiting effects were caused by the NC miR mimic (Fig. 3a). These results were additionally supported by Western blot analysis in SaOS-2 cells transiently transfected with miR-218 mimic, which revealed a substantial decline in RUNX2 protein accumulation due to forced elevation of miR-218 (Fig. 3b and Fig. S1), and a weak reverse effect was observed in case of antimiR-218 mimic transfection.

Next, we engineered miR reporter plasmids for the putative recognition sites of $M E F 2 C$ and COL1OA1 3' UTRs. The luciferase activity for the wild-type $M E F 2 C$ reporter was significantly reduced by the miR-218 mimic, while non-targeting (NC) miR mimic failed to reduce the reporter activity. A direct targeting effect of miR-218 mimic for $M E F 2 C$ was additionally confirmed using mutated 3'UTR seed sequence for the $M E F 2 C$ reporter (Fig. 3c). Again, this was verified in SaOS-2 cells transiently transfected either with miR-218 mimic, or with anti-miR-218, when decreased accumulation of MEF2C protein was observed due to a gain of miR-218 levels, and this effect went to the opposite direction upon transfection with anti-miR-218 (Fig. 3d and Fig. S1).

A similar inhibiting effect on luciferase activity was seen for a reporter containing a COL10A1 3'UTR with a

Table $\mathbf{2}$ In silico analysis for annealing efficiencies between putative mRNA targets and miR-218

\begin{tabular}{|c|c|c|c|c|}
\hline $\begin{array}{l}\text { Name of } \\
\text { miR }\end{array}$ & $\begin{array}{l}\text { Putative } \\
\text { Targets }\end{array}$ & $\begin{array}{l}\text { Predicted pairing of miR (top) } \\
\text { and mRNA (bottom) }\end{array}$ & $\begin{array}{l}\text { mirSVR } \\
\text { score } \\
\end{array}$ & $\begin{array}{l}\text { Position at } \\
\text { 3'UTR }\end{array}$ \\
\hline miR-218 & $R U N X 2$ & $\begin{array}{c}\text { 3'uguaccaaucuaguucguguu5' } \\
|||||||||| \mid \\
\text { 5'uauuaaa-aga-caagcacaa3، }\end{array}$ & -0.6329 & $1296-1316$ \\
\hline & $M E F 2 C$ & 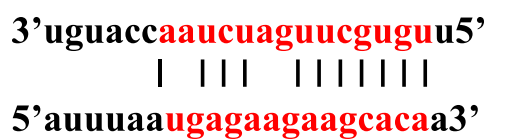 & -0.8156 & $2847-2868$ \\
\hline & COL10A1 & $\begin{array}{l}\text { 3'uguaccaaucuaguucguguu5' } \\
\text { III I I I IIIII } \\
\text { 5'ccucacuua-uuaaagcacaa3' }\end{array}$ & -1.2533 & $978-998$ \\
\hline
\end{tabular}


a

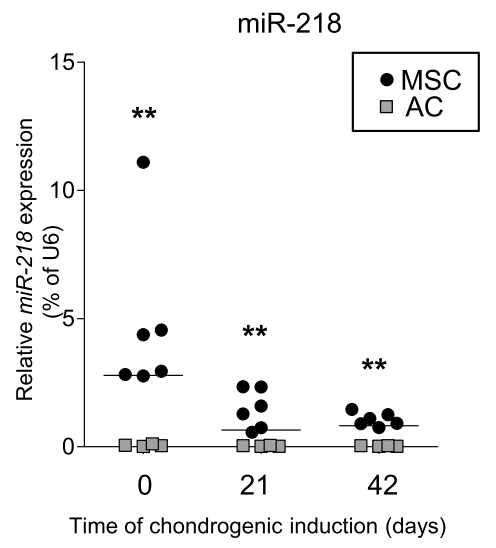

b

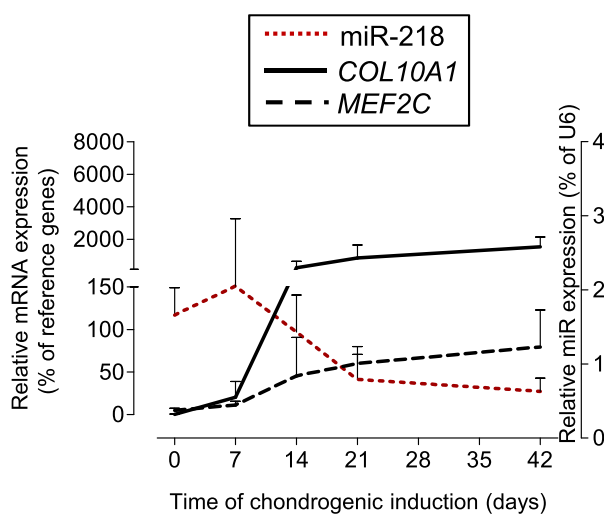

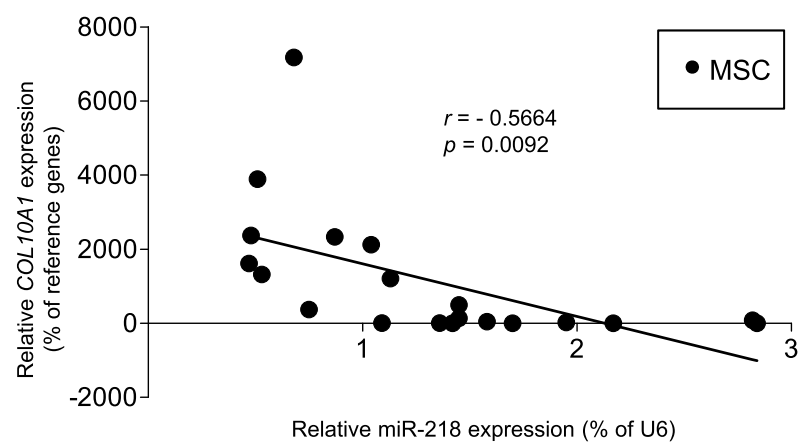

Fig. 2 Negative correlation for gene expression kinetics between miR-218 and MEF2C or COL1OA1 in MSC during chondrogenic differentiation. a Differential expression of miR-218 between MSC ( $N=6$ donors, black circles) and AC ( $N=4$ donors, gray squares) was monitored at indicated time points during chondrogenic differentiation by qRT-PCR; vertical lines correspond to median values; ${ }^{*} p \leq 0.01$ (Mann-Whitney $U$ ). b MSC ( $N=4$ donors) were subjected to chondrogenic induction, and expression of MEF2C (black-dotted line) and COL10A1 (black line) mRNA and miR218 (red-dotted line) was measured by qRT-PCR at indicated time points. Mean values \pm SD are shown. c Correlation between COL10A1 and miR218 expression levels in MSC ( $N=4$ donors) was measured by qRT-PCR. Pearson correlation coefficient with its $p$ value is indicated

putative seed sequence for miR-218 (Fig. 3e). Furthermore, in SaOS-2 cells, gain of miR-218 reduced COL1OA1 expression, although only by a trend due to very low endogenous levels of COL1OA1 mRNA in SaOS-2 cells (Fig. 3f and Fig. S1).

Collectively, these data indicated that miR-218 targets and downregulates a number of molecules involved in the endochondral pathway, including RUNX2, MEF2C, and COL1OA1. Thus, it could be expected that known downstream targets of MEF2C and RUNX2 transcription factors, like ALPL and IBSP genes, might also be affected, although they are not direct targets of miR-218. Indeed, in SaOS-2 cells, the gain of miR-218 resulted in significant reduction of ALP activity (Fig. 4a). Moreover, IBSP expression significantly dropped as well (Fig. $4 \mathrm{~b}$ ). $I B S P$ is the major structural protein of the bone matrix, and its elevated expression is also associated with hypertrophy. In human cells, it has been shown to be regulated along with MEF2C, and not affected by RUNX2 [20].Therefore, miR-218 might regulate a whole cascade of hypertrophy-related factors during MSC chondrogenesis. To address this important question, we next assessed whether the effects caused by miR-218 in the osteosarcoma cell line model (SaOS-2) could be translated into human MSC during a course of chondrogenic differentiation.

\section{MiR-218 targets hypertrophic markers in MSC}

To address the functional role of miR-218 in human MSC, we conducted a miR pulldown assay for a direct detection of miR-218 mRNA targets (Fig. 5). For this, miR-218 mimic labeled with biotin was incubated with the cytosolic fraction of a cellular extract of expanded human MSC, and using magnetic streptavidin beads, immobilized mRNA targets were isolated, purified, and analyzed. For the tested targets of miR-218: $M E F 2 C$ and RUNX2, we detected high levels of enrichment in comparison to a non-targeting $\mathrm{NC}$ control miR mimic. COL10A1 remained untested since it is not expressed at day 0 of chondrogenesis in MSC. Interestingly, although 
Table 3 Significantly enriched KEGG pathways associated with the predicted target genes for miR-218 (selected categories for "signal transduction," "signaling molecules and interaction," and "glycan biosynthesis and metabolism" identifiers)

\begin{tabular}{lll}
\hline KEGG pathway & $\begin{array}{l}\text { Number of } \\
\text { genes }\end{array}$ & $\boldsymbol{P}$ value \\
\hline PI3K-Akt signaling pathway & 106 & $3.7 \mathrm{E}-3$ \\
MAPK signaling pathway & 86 & $3.5 \mathrm{E}-4$ \\
CAMP signaling pathway & 72 & $1.2 \mathrm{E}-4$ \\
Calcium signaling pathway & 67 & $7.9 \mathrm{E}-5$ \\
Hippo signaling pathway & 56 & $4.3 \mathrm{E}-4$ \\
AMPK signaling pathway & 49 & $1.6 \mathrm{E}-4$ \\
Signaling pathways regulating & 45 & $2.9 \mathrm{E}-2$ \\
pluripotency of stem cells & & \\
Wnt signaling pathway & 43 & $5.3 \mathrm{E}-2$ \\
Thyroid hormone signaling pathway & 40 & $1.1 \mathrm{E}-2$ \\
HIF-1 signaling pathway & 38 & $1.1 \mathrm{E}-3$ \\
TNF signaling pathway & 35 & $4.3 \mathrm{E}-2$ \\
NF-kappa B signaling pathway & 30 & $3.3 \mathrm{E}-2$ \\
ECM-receptor interaction & 28 & $8.6 \mathrm{E}-2$ \\
mTOR signaling pathway & 27 & $4.3 \mathrm{E}-4$ \\
TGF-beta signaling pathway & 27 & $9.3 \mathrm{E}-2$ \\
Hedgehog signaling pathway & 11 & $9.3 \mathrm{E}-2$ \\
Glycosaminoglycan biosynthesis & 9 & $8.6 \mathrm{E}-2$ \\
- chondroitin sulfate/dermatan sulfate & & \\
\hline
\end{tabular}

ROBO1 (Roundabout 1) was earlier reported as a miR218 target in rheumatoid arthritis (RA)-fibroblast-like synovial cells (FLS) [43], in human MSC, no interaction between miR-218 and ROBO1 was detected (Fig. 5). Similarly, there was no enrichment for HPGD that earlier was reported to be a direct target of miR-218 in rabbit synovium-derived MSC [30] (data not shown). Collectively, these data emphasize that specific mRNA targets of miR-218 might also depend on a particular cellular context or a selected species.

\section{Effect of miR-218 on chondral versus endochondral differentiation of MSC}

To investigate whether miR-218 affects hypertrophy during MSC chondrogenesis, we transfected the cells with miR-218 mimic and followed-up the expression levels of hypertrophic markers during 28 days of a chondrogenic differentiation time course (Fig. 6 and Fig. S2). First, we confirmed that significantly elevated levels of miR-218 mimic persist over the 28 days' time course of chondrogenic differentiation (Fig. S2). This also caused significant reduction of RUNX2 expression by day 21 in MSC transfected with miR-218 in comparison to $\mathrm{NC}$ mimic control (Fig. 6). We also found that expression levels of $M E F 2 C, C O L 10 A 1$, and $A L P L$ were decreased by the trend by day 21, with no apparent changes in COL2A1 gene expression. Remarkably, these effects were accompanied by significant elevation of $M M P 3$ expression, indicating that although miR-218 caused reduction of several hypertrophic markers, a feedback loop might have been activated leading to induction of metalloproteinase MMP3 expression (Fig. 6).

Having observed some inhibitory effects on hypertrophy by gain of miR-218, we were also interested to follow whether progression of chondrogenesis changed. Safranin O staining revealed only a slight trend for less proteoglycan deposition by MSC-derived chondrocytes at day 28 post-transfection with miR-218 in comparison to the non-targeting (NC) mimic transfection control (Fig. S3a). No apparent changes in GAG/DNA content were observed in both groups, with only a slight trend for reduced glycosaminoglycan deposition in case of MSC transfected with miR-218 (Fig. S3b).

Collectively, these data suggest that, although miR-218 had the ability to target the tested hypertrophic markers, it did not result either in significant reduction of the hypertrophic phenotype, or in an additional boost of chondrogenesis. Thus, no positive effect on a transition from endochondral towards chondral outcome during MSC chondrogenesis could be achieved when only this single miR was manipulated.

Since it has been established before that regulation of both MEF2C [20] and RUNX2 [44] is WNT/ $\beta$-catenin signaling-dependent, we were interested to find out whether ectopic overexpression of miR-218 might also cause alterations to WNT signaling. Indeed, we found that gain of miR-218 resulted in accumulation of active cytosolic $\beta$-catenin by day 28 of MSC chondrogenesis (Fig. 7), thus indicating that miR-218 might also target some of the WNT signaling inhibitors. According to an earlier report, miR-218 could activate canonical WNT signaling in mouse cells due to targeting DKK2 and SFRP2 [45]. Both DKK2 and SFRP2 are modulators of WNT signaling and could function as agonists or antagonists, depending on the cellular context and the presence of their co-factors (e.g., Kremen 2, in case of DKK2) $[46,47]$. It is known that $\mathrm{WNT} / \beta$-catenin signaling largely contributes to MSC hypertrophy [11]. Therefore, miR-218 may not only inhibit hypertrophic markers but also induce a feedback loop that restores hypertrophy due to concomitant activation of $\mathrm{WNT} / \beta$ catenin signaling.

\section{Expression of miR-218 in cartilage of OA patients}

Finally, as hypertrophic degeneration of chondrocytes is one of the major characteristics of OA development, we hypothesized that miR-218 might be differentially expressed between healthy and pathological phenotypes of cartilage tissue. We assessed expression levels of miR- 
a

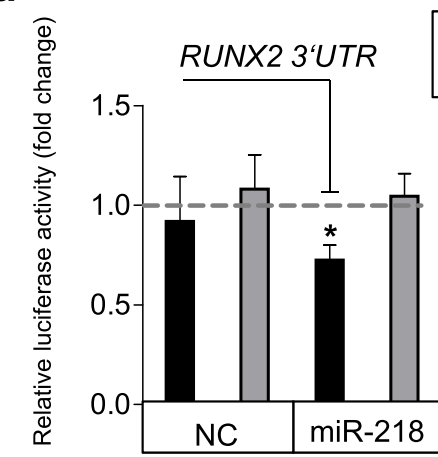

C
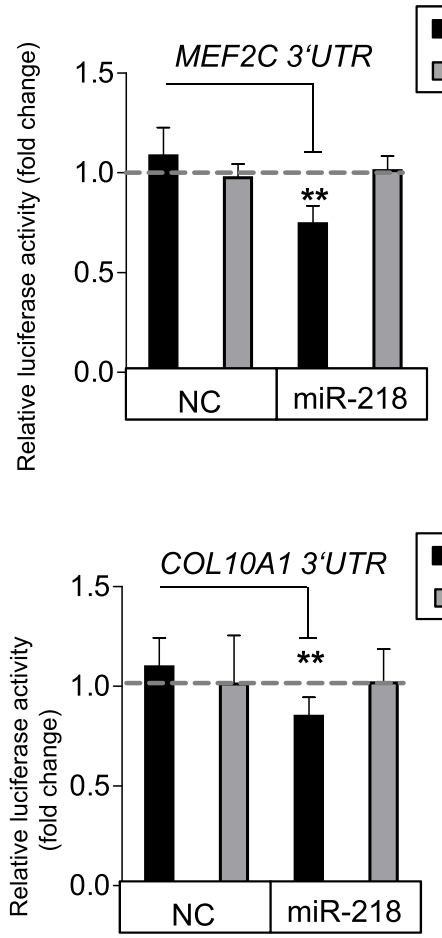

b

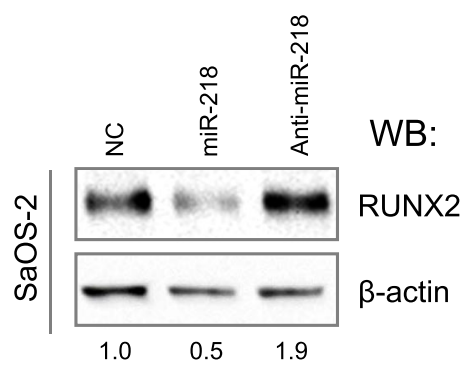

d

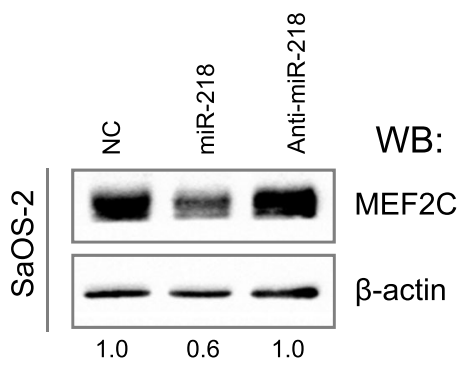

f

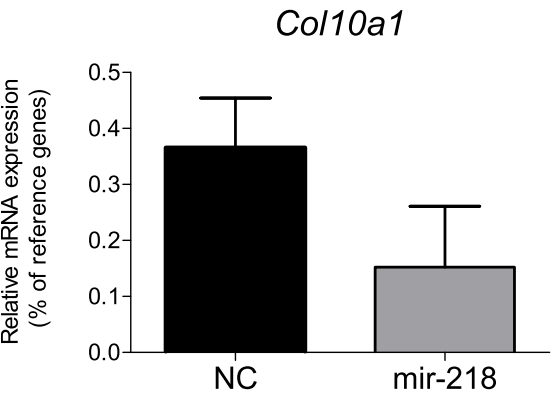

Fig. 3 Hypertrophic marker genes are targets of miR-218. a, c, e): Luciferase assay in HEK293T cells conducted with pMIR-REPORT vector either containing wild-type (wt, black) or mutated (gray) 3'-UTR fragment of RUNX2 (a), or MEF2C (c), or COL10A1 (e) with a putative binding-side for miR-218. Non-targeting control miR mimic (NC) was used as a control. Gray-dotted lines indicate levels of binding for an empty pMIR-REPORT vector; $n=3$. b, $\mathbf{d}$, $\mathbf{f}$ SaOS-2 cells were transiently transfected with either miR-218, or with anti-miR-218 with non-targeting control (NC) mimics, and abundancies of RUNX2 (b) or MEF2C (d) proteins were detected by WB (numbers below indicate fold changes in comparison to NC control, $n=3$ (RUNX2) or 5 (MEF2C)), or COL10A1 mRNA expression was measured by qRT-PCR, $n=2$ (f). Mean values \pm SD are shown. ${ }^{* *} p \leq 0.01$

(Mann-Whitney $U$ )

218 in healthy donors and OA patients (Fig. 8). It is important to mention that all the cartilage samples were additionally stratified by levels of miR-675 expression, a reliable marker of OA progression in our hands [37, 48]. Indeed, healthy and $\mathrm{OA}$ patient cohorts were wellsegregated due to significantly different levels of miR675 expression. However, no significant differences between chondrocytes from healthy donors and OA patients were observed for miR-218 expression. Thus, in our tested cohorts, we could not confirm a conclusion made by a previous report claiming that miR-218 is a prognostic marker and a therapeutic target for OA treatment [49].

Altogether, our data suggest that anti-hypertrophic miR-218 affects a number of important genes within a hypertrophic differentiation cascade (Fig. 9). While miR218 targeted hypertrophy-related mRNAs, such as $M E F 2 C$ and its downstream targets COL1OA1 and IBSP, as well as $R U N X 2$, a transcription factor regulating the expression of COL1OA1 and ALPL, it also resulted in 


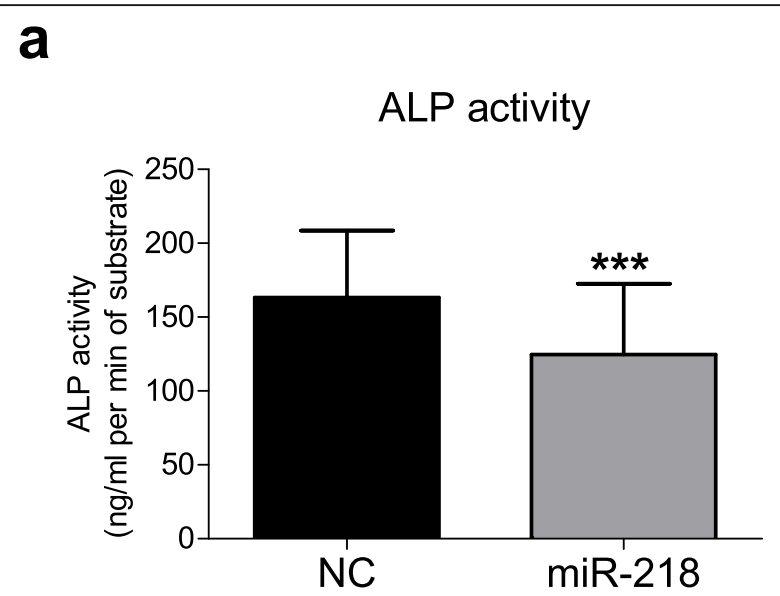

b

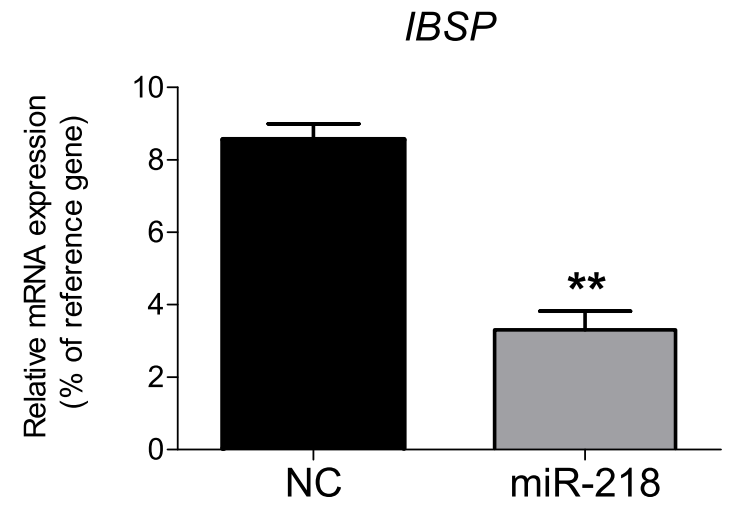

Fig. 4 miR-218 reduces hypertrophic markers in SaOS-2 cells. SaOS-2 cells were transiently transfected with miR-218 mimic (gray) or with a non-targeting control miR mimic (NC, black), and ALP activity was measured in cell culture supernatants $48 \mathrm{~h}$ later $(n=8)$ (a mean values \pm SEM are shown), or expression of IBSP MRNA was monitored by qRT-PCR $(n=3)$ ( $\mathbf{b}$ mean values \pm SD are shown); ${ }^{* *} p \leq 0.01$ and ${ }^{* *} p \leq 0.001$; (two-tailed paired $t$ test) accumulation of $\beta$-catenin. $\mathrm{WNT} / \beta$-catenin signaling was induced, probably, due to a concomitant targeting of WNT signaling inhibitors, such as SFRP2 and DKK2, which in turn led to the activation of a feedback loop counteracting the anti-hypertrophic effects of miR-218. Therefore, one cannot exclude that other mRNA targets regulated by miR-218 might influence the final outcome of chondral versus endochondral progression, depending on a particular cellular context.

\section{Discussion}

The cartilage tissue is characterized by a low cellular density and vascular components, and it is mostly comprised by proteoglycans and extracellular collagens. It has a very limited capacity to heal in case of injury or degeneration processes due to osteoarthritis. Therefore, therapeutic approaches with application of alternative cells sources, such as MSC that are capable of chondrogenic differentiation, are at a focus of orthopedic research. However, a long-standing problem with application of MSC for cartilage regeneration is their tendency to undergo hypertrophic degeneration that could lead to endochondral bone induction after transplantation. The most characteristic feature of hypertrophic chondrocytes is expression of type $\mathrm{X}$ collagen and ALP. These markers are absent in articular chondrocytes that retain the stable phenotype. This implies that the hypertrophic pathways promoted in MSC during chondrogenesis are completely blocked in AC due to some yet to be identified molecular mechanisms.

There is growing evidence that microRNAs are involved in chondrogenic differentiation of MSC. However, there are only a limited number of studies on a regulatory role of miRs in hypertrophy. To address a

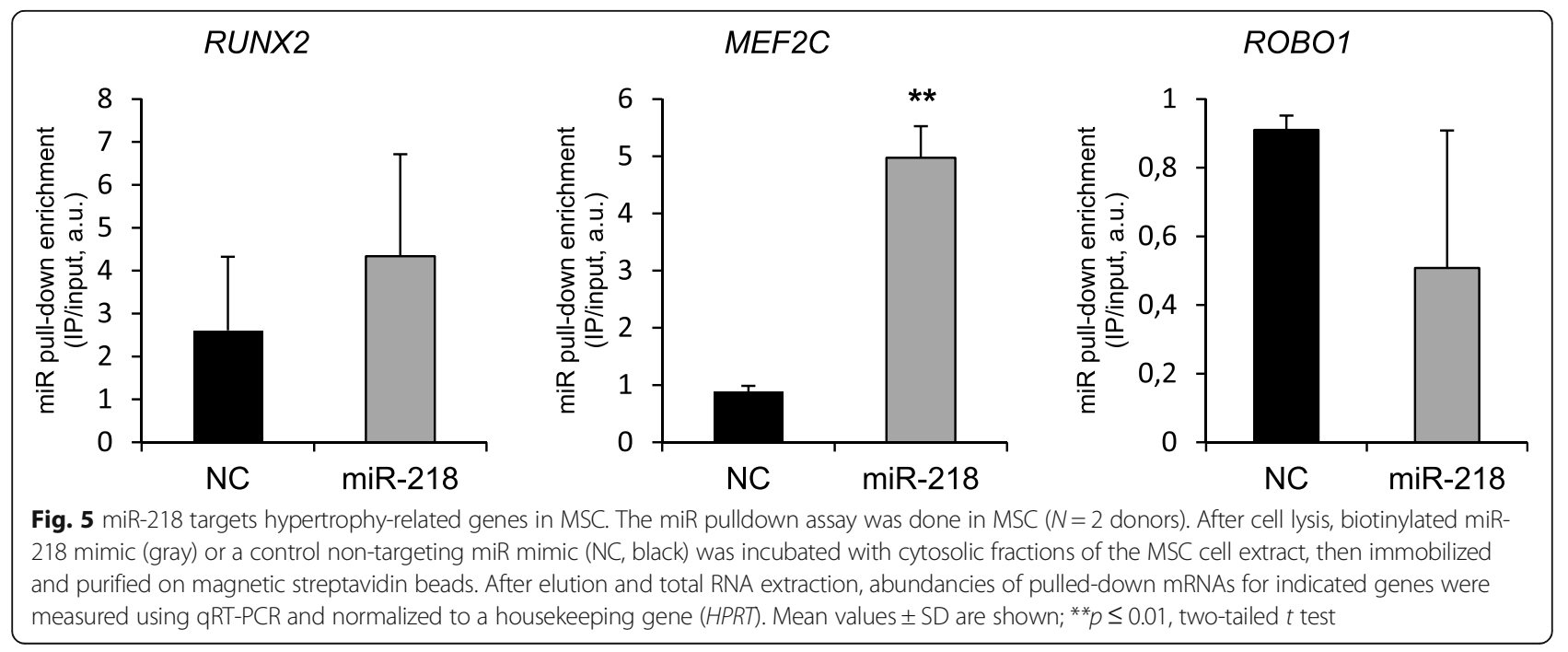




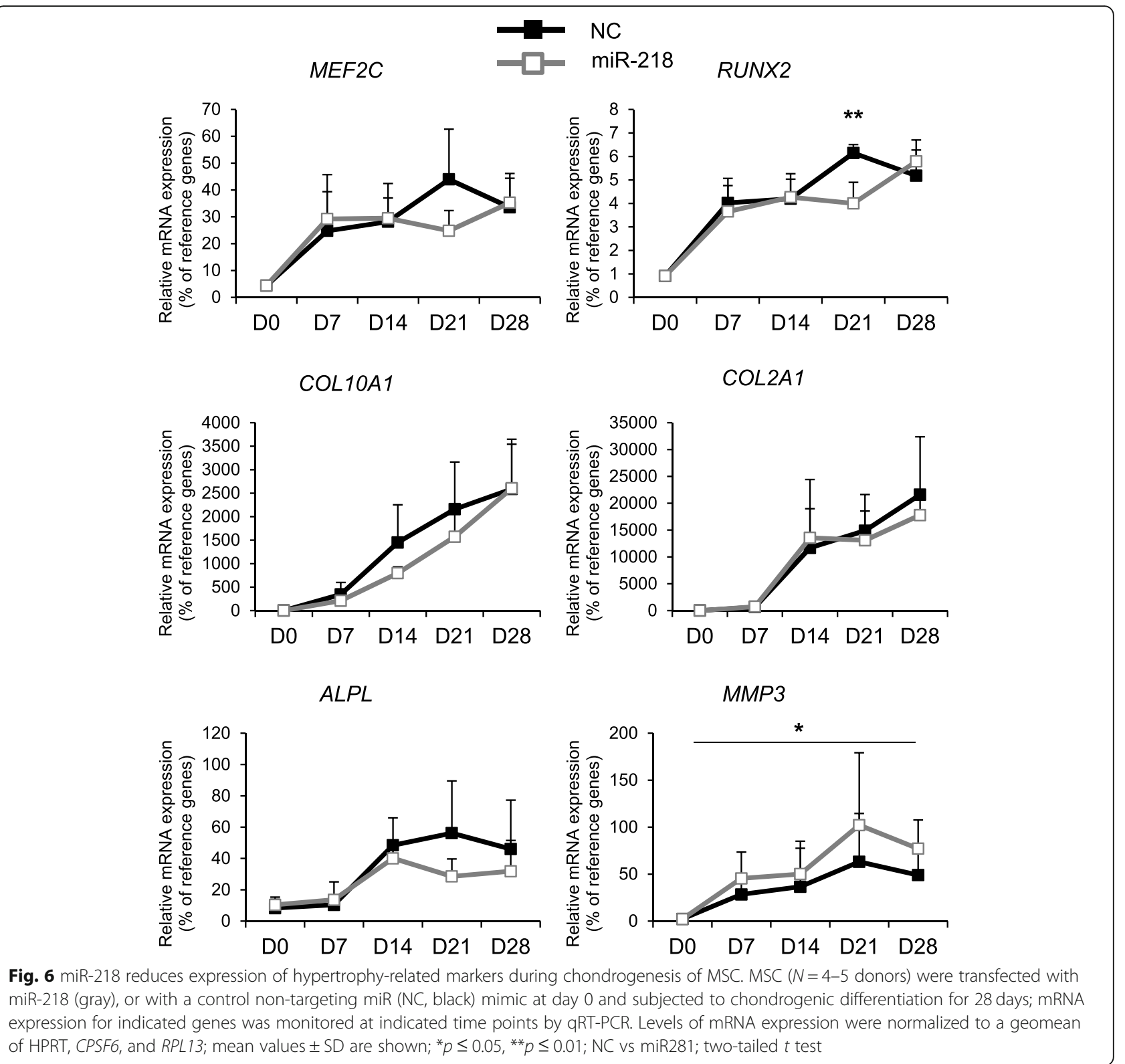

question about miRs' role in driving hypertrophy, we conducted a comparative analysis of expression profiles for 1349 miRs between hypertrophic and articular chondrocytes. Our focus was on finding characteristic signature-miRs that would define these two different chondrocyte phenotypes. Among the top 15 differentially expressed miRs, we selected miR-218 that resembled a suitable candidate, as among its in silico predicted targets, there were well-characterized hypertrophy markers, such as MEF2C, RUNX2, and COL1OA1.

Mir-218-5p is a microRNA found to be expressed in 20 vertebrate species (http://www.mirbase.org/). It has been implicated in tumorigenesis and cancer progression of different tumor entities (reviewed in [50]), as well as its role in the motor neuron development has recently been discovered [51, 52]. However, regarding the role of miR-218 in chondrogenesis, the published literature is very limited and so far presents somewhat contradicting claims. Namely, there is a single study conducted with application of rabbit synovium-derived MSC that concluded the prochondrogenic role of miR-218 in early chondrogenesis due to targeting $H P G D$, although a link between HPGD alterations and effects on chondrogenesis has not been established [30]. On the other hand, another study implied that elevated expression of miR-218 
a

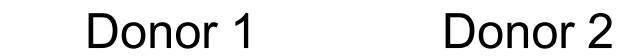

NC $\quad$ miR-218 NC miR-218

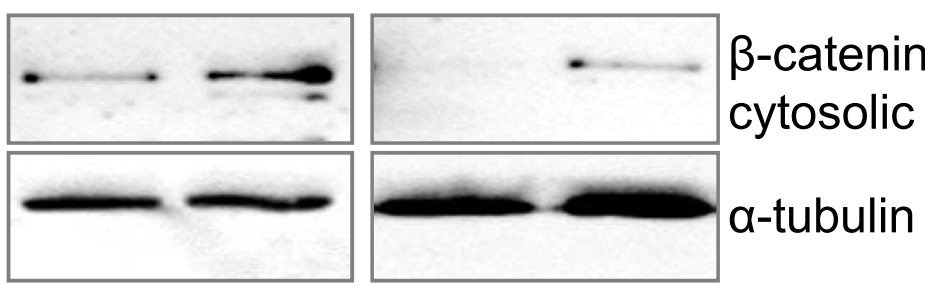

Donor 3 Donor 4

NC $\quad$ miR-218 NC miR-218

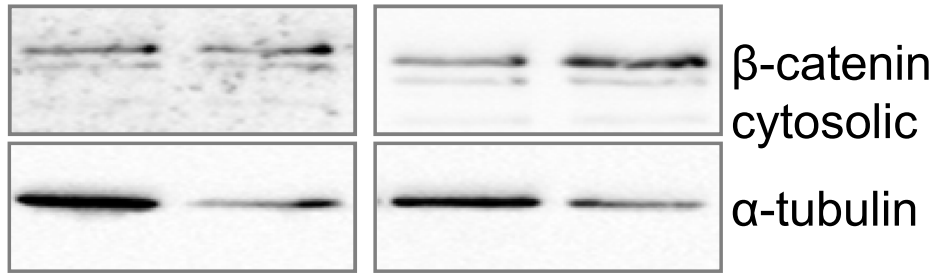

b

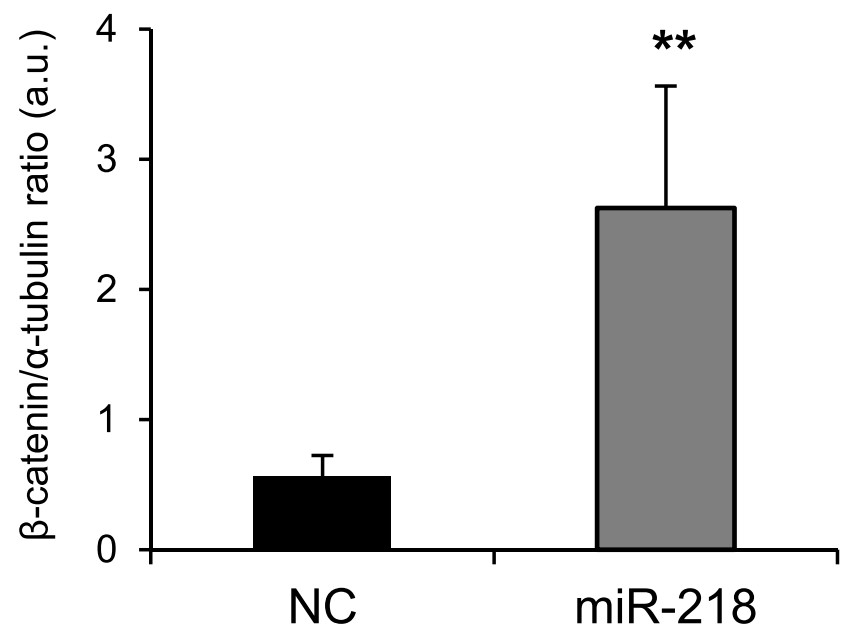

Fig. 7 Gain of miR-218 at the start of chondrogenic differentiation leads to increased accumulation of cytosolic $\beta$-catenin. MSC ( $N=4$ donors) were transfected with miR-218 (gray), or with a control non-targeting miR (NC, black) mimic at day 0 , subjected to chondrogenic differentiation for 28 days, and $\beta$-catenin accumulation (a) was assessed by Western blot analysis at day 28. b Semi-quantitative analysis of WB images; $\mathbf{a}$-tubulin was used as a loading control; mean values $\pm \mathrm{SD}$ are shown; $N=4$; ${ }^{*} p \leq 0.01$, two-tailed $t$ test

positively correlates with $\mathrm{OA}$ progression and cartilage tissue loss in patients due to targeting PIK3C2A of Akt pathway, thus contradicting a conclusion of the study mentioned above about pro-chondrogenic effects of miR-218 [49]. To summarize, no studies on the role of miR-218 in human MSC that would question whether there is a direct link between miR218 and chondrocyte hypertrophy have been conducted to date.

It has been already found that miR-218 could target Runx2 in mouse ACDT5 cell line, although this effect was found to be cell-type dependent [28]. 


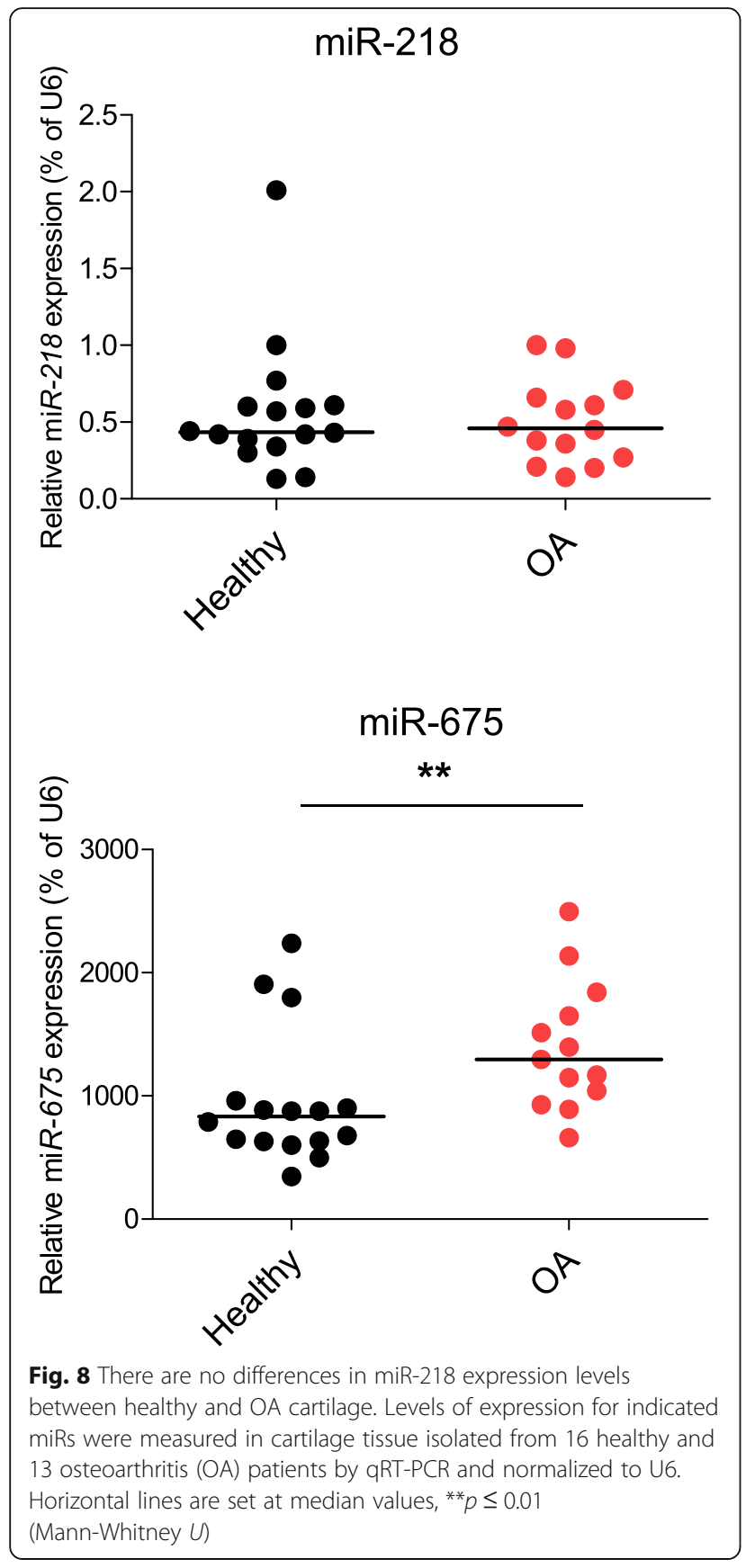

Nevertheless, we used this finding as a positive example for evaluation of other putative targets of miR218, MEF2C and COL1OA1, and confirmed that all three genes were directly affected by an ectopic miR218 mimic, as the corresponding reporters' activity, gene expression, and protein accumulation were reduced. Since miR-218 was found to regulate not only one target related to hypertrophy but a number of genes of the hypertrophic cascade, including those that were not predicted to directly interact with miR-
218, such as $I B S P$ and $A L P L$, we concluded an antihypertrophic role of miR-218.

An important question, however, remained whether miR-218 could not only prevent hypertrophy but also promote chondral differentiation of human MSC as one might expect when the expression of anti-chondrogenic RUNX2 [20] is reduced. Although we were able to confirm targeting of hypertrophic markers by miR-218 in human MSC, we did not achieve any significant antihypertrophic effects, as well as a hypothesis about its pro-chondrogenic role had to be rejected, as additional gain of miR-218 at start of differentiation failed to boost chondrogenic markers further. These results contradict the previous report about the ability of miR-218 to promote early chondrogenesis of rabbit MSC from synovial fluid [30]. However, we also argue against a suggested pro-osteogenic role of miR-218 due to targeting of ROBO1 reported for rheumatoid arthritis FLS [43]. Indeed, we did not detect interaction between $R O B O 1$ and the miR-218 bait in MSC, probably, due to different representation of multiple putative mRNA targets present at a given cell type. It also implies that the same miR might play different regulatory functions depending on a particular cellular context. We also found that, although miR-218 reduced hypertrophic mRNAs by trend, it may also target genes that in turn induce pro-hypertrophic pathways, like $\mathrm{WNT} / \beta$ catenin signaling, thus generating a feedback loop that prevents a shift towards a stable chondrogenic phenotype. Therefore, to achieve a full inhibition of the endochondral pathway and successful outcome of chondral development of human MSC, not one, but a whole class of anti-hypertrophic miRs, including miR218, needs to be taken into consideration for tissue engineering purposes. Finding these additional factors that are able to control chondrogenic differentiation and prevent hypertrophy would be of interest in future investigations.

Next, we also speculated that miR-218 might serve as a marker to distinguish between the healthy and pathological phenotype of chondrocytes in OA, as the upregulation of hypertrophic markers in chondrocytes is one of the main characteristics of OA cartilage [37, 53]. In contrary to a recent report suggesting that an upregulation of miR-218 is a characteristic of cartilage destruction in OA patients [49], no substantial differences between healthy and OA cartilages were found for miR-218 within our experimental cohorts. Importantly, for stratification of cartilage samples, we additionally applied expression of miR-675 a decisive factor to verify segregation of healthy and OA cohorts, as miR-675 has been reported to be a reliable marker for OA progression [37, 48]. Nevertheless, even without this stratification, in our larger samples 


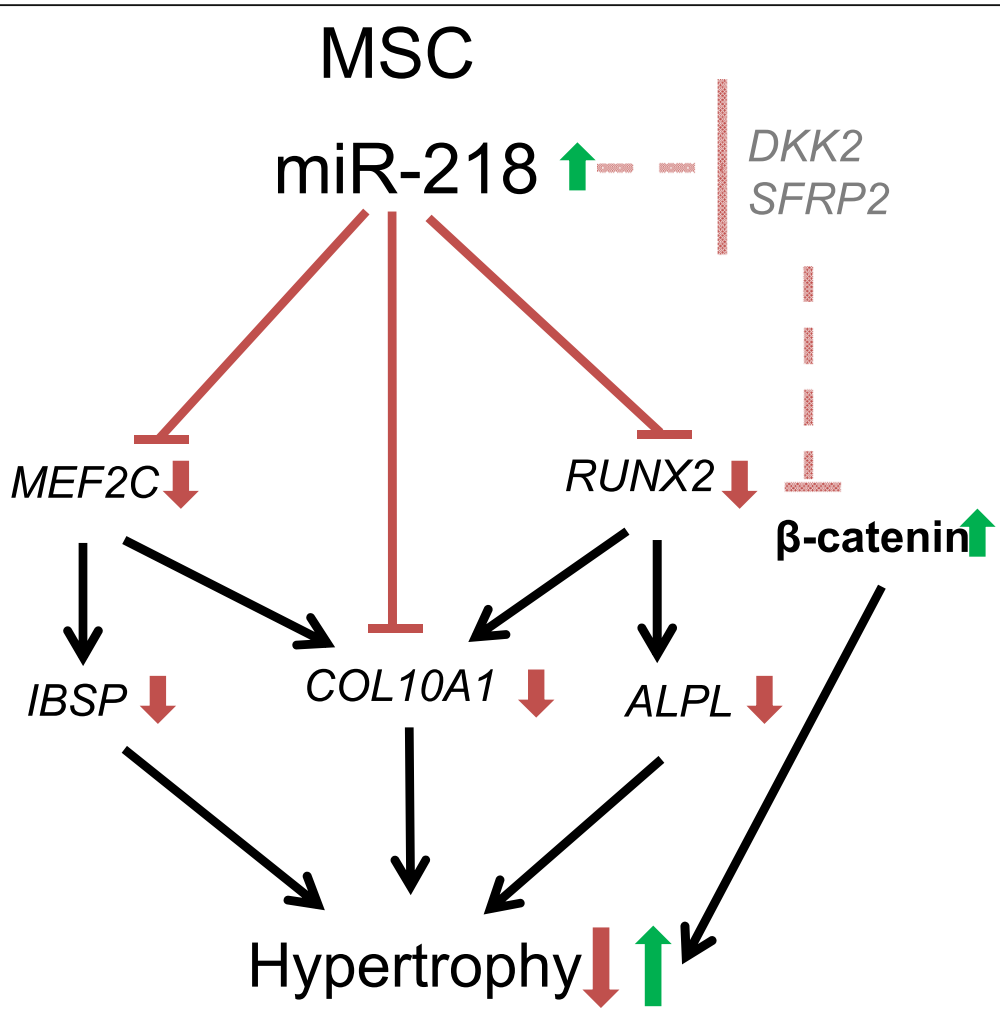

Fig. 9 A scheme depicting a putative mechanism for the anti-hypertrophic effects caused by miR-218 in MSC and activation of a feedback loop due to concomitant effects on inhibitors of WNT/B-catenin signaling. MiR-218 targets hypertrophy-related mRNAs: MEF2C and its downstream targets, COL10A1 and IBSP, leading to attenuation of the hypertrophic phenotype. Among the other targets of miR-218, there is RUNX2, a transcription factor regulating expression of COL1OA1 and ALPL. However, accumulation of $\beta$-catenin due to concomitant targeting of WNT signaling inhibitors activates a feedback loop that counteracts the anti-hypertrophic alterations. Arrows indicate directions of expression regulation: red—downregulation, green —upregulation

sets, no differential expression between healthy donors and OA patients cohort was found. This highlights that a conclusion about miR-218 as a prognostic marker and a therapeutic target for OA treatment has to be taken with precaution.

\section{Conclusions}

In conclusion, our study suggests that miR-218 contributes to a balance between endochondral versus chondral differentiation in human MSC as it targets a number of important genes regulating the hypertrophic pathway. While miR-218 suppresses $M E F 2 C$, RUNX2, and COL1OA1 that define the terminal differentiation of chondrocytes, it may also reduce other genes inhibiting the pro-hypertrophic $\mathrm{WNT} / \beta$-catenin pathway, thus activating a feedback loop. Therefore, with a single miR, one cannot achieve a challenging goal of a phenotype switch from endochondral to chondral, and a whole class of hypertrophy-regulating miRs have to be taken into consideration. These findings give new insights into mechanisms of hypertrophic differentiation and chondrogenesis in human MSC and offer a perspective for further investigations into understanding mechanisms regulating cartilage disorders.

\section{Supplementary Information}

The online version contains supplementary material available at https://doi. org/10.1186/s13287-020-02026-6.

Additional file 1: Supplementary Table S1. List of qRT-PCR primers used in this study. Supplementary Table S2. List of cloning primers used in this study. Fig. S1. miR-218 transfection efficiency in SaOS-2 cells. SaOS-2 cells were transiently transfected with miR-218 (grey), or with non-targeting control (NC, black) mimic, and transfection efficiency was controlled by qRT-PCR. Mean values \pm SD are shown, $n=2 ;{ }^{* * *} p \leq 0.001$, two-tailed t-test. Fig. S2. miR-218 transfection efficiency in human MSC. MSC cells ( $N=3$ donors) were transiently transfected with miR-218 (grey), or with non-targeting control (NC, black) mimic before cells were subjected to chondrogenic differentiation for 28 days, and transfection efficiency was controlled by qRT-PCR at indicated time points. Mean values $\pm \mathrm{SD}$ are shown; ${ }^{*} p \leq 0.05$, NC vs miR-218; expression kinetic curves were compared using two-tailed paired t-test. Fig. S3. Gain of function of miR-218 at the start of chondrogenic differentiation does not additionally boost MSC chondrogenesis. MSC ( $N=4$ - 6) were transfected with miR218 (grey), or with a control non-targeting miR (NC, black) mimics at day 0 , and subjected to chondrogenic differentiation for 28 days. a, Safranin O staining of $3 \mathrm{D}$ pellet cultures at day 28 chondrogenesis. Scale bar, 500 $\mu \mathrm{m}$. b, Total GAG and DNA content of 3D pellet cultures was assessed by DMMB and PicoGreen assays at day 28 of chondrogenesis; Mean values $\pm \mathrm{SD}$ are shown; $N=6$ donors. 


\section{Abbreviations}

AC: Articular chondrocytes; ATCC: American Type Culture Collection; ALP: Alkaline phosphatase; BMP: Bone morphogenetic protein; DAVID: The Database for Annotation, Visualization and Integrated Discovery; DSC: Dental stem cells; DMMB: Dimethyl-methylene blue;

EDTA: Ethylenediaminetetraacetic acid; FCS: Fetal calf serum; FGF: Fibroblast growth factor; GO: Gene ontology; HBV: Hepatitis B virus; HCV: Hepatitis C virus; HIV: Human immunodeficiency virus; IGF: Insulin-like growth factor; IHH: Indian hedgehog; MAD: Median of absolute deviation; MSC: Mesenchymal stromal cells; miR: MicroRNA; OA: Osteoarthritis; qRTPCR: Quantitative reverse-transcriptase polymerase chain reaction: PBS: Phosphate-buffered saline; PTHrP: Parathyroid hormone-related protein; mirSVR: microRNA score of vector regression; TGF $\beta$ : Transforming growth factor beta; 3'UTR: 3'-Untranslated region; WB: Western blotting

\section{Acknowledgements}

The authors thank Tanja Sims for technical assistance and Simone Gantz for advise with statistical analysis of the study. The authors thank the patients for donation of their cells and physicians for coordination of the sample provision. Support by the Genomics Core Facility at German Cancer Research Centre (DKFZ, Heidelberg, Germany) provided for microarray analysis is gratefully acknowledged.

\section{Authors' contributions}

WR conceived, designed, and supervised the project. SM, JG, SID, NHe, and NHo conducted all experiments and processed and analyzed data. TG provided administration and provision of study materials from patients. SM and WR wrote the manuscript. The authors discussed the data and read, commented, and approved the manuscript.

\section{Funding}

This study was financially supported by the German Research Foundation (DFG2/707/12-1). Open Access funding enabled and organized by Projekt DEAL.

\section{Availability of data and materials}

The datasets generated during and/or analyzed during the current study are available from the corresponding author on reasonable request.

\section{Ethics approval and consent to participate}

The study was approved by the local ethics committee (Medical Faculty of the University of Heidelberg), and informed consent was obtained from all the patients participating in the study, according to the 1964 Declaration of Helsinki, updated in 2000.

\section{Consent for publication}

All authors approved the manuscript, and their written informed consent for the publication was obtained.

\section{Competing interests}

The authors declare that there is no conflict of interest regarding the publication of this article.

\section{Author details}

${ }^{1}$ Research Centre for Experimental Orthopaedics, Heidelberg University Hospital, Heidelberg, Germany. ${ }^{2}$ Clinic for Orthopaedics and Trauma Surgery, Heidelberg University Hospital, Heidelberg, Germany.

\section{Received: 19 August 2020 Accepted: 13 November 2020}

Published online: 10 December 2020

\section{References}

1. Richardson SM, et al. Mesenchymal stem cells in regenerative medicine: focus on articular cartilage and intervertebral disc regeneration. Methods. 2016;99:69-80

2. Xian CJ, Foster BK. Repair of injured articular and growth plate cartilage using mesenchymal stem cells and chondrogenic gene therapy. Curr Stem Cell Res Ther. 2006; (2):213-29.

3. Pelttari $\mathrm{K}$, et al. Premature induction of hypertrophy during in vitro chondrogenesis of human mesenchymal stem cells correlates with calcification and vascular invasion after ectopic transplantation in SCID mice. Arthritis Rheum. 2006;54(10):3254-66.

4. Pelttari K, Steck E, Richter W. The use of mesenchymal stem cells for chondrogenesis. Injury. 2008;39(Suppl 1):S58-65.

5. Dreier R. Hypertrophic differentiation of chondrocytes in osteoarthritis: the developmental aspect of degenerative joint disorders. Arthritis Res Ther. 2010;12(5):216.

6. Weiss $\mathrm{S}$, et al. Impact of growth factors and PTHrP on early and late chondrogenic differentiation of human mesenchymal stem cells. J Cell Physiol. 2010;223(1):84-93.

7. Longobardi L, et al. Subcellular localization of IRS-1 in IGF-I-mediated chondrogenic proliferation, differentiation and hypertrophy of bone marrow mesenchymal stem cells. Growth Factors. 2009;27(5):309-20.

8. Fischer J, et al. Role of PTHrP(1-34) pulse frequency versus pulse duration to enhance mesenchymal stromal cell chondrogenesis. J Cell Physiol. 2016; 231(12):2673-81.

9. Yoshida CA, et al. Runx2 and Runx3 are essential for chondrocyte maturation, and Runx2 regulates limb growth through induction of Indian hedgehog. Genes Dev. 2004;18(8):952-63.

10. Weiss $S$, et al. Reduction of hypertrophy by PTHrP and bFGF during in vitro chondrogenesis of mesenchymal stem cells from bone marrow. J Stem Cells Regen Med. 2007;2(1):31-2.

11. Diederichs S, Tonnier V, März M, Dreher SI, Geisbüsch A, Richter W. Regulation of WNT5A and WNT11 during MSC in vitro chondrogenesis: WNT inhibition lowers BMP and hedgehog activity, and reduces hypertrophy. Cell Mol Life Sci. 2019;76(19):3875-89. https://doi.org/10.1007/ s00018-019-03099-0. Epub 2019 Apr 12. PMID: 30980110.

12. Dexheimer V, et al. Differential expression of TGF-beta superfamily members and role of Smad1/5/9-signalling in chondral versus endochondral chondrocyte differentiation. Sci Rep. 2016;6:36655.

13. Arnold MA, et al. MEF2C transcription factor controls chondrocyte hypertrophy and bone development. Dev Cell. 2007:12(3):377-89.

14. Takeda $\mathrm{S}$, et al. Continuous expression of $\mathrm{Cbfa} 1$ in nonhypertrophic chondrocytes uncovers its ability to induce hypertrophic chondrocyte differentiation and partially rescues Cbfa1-deficient mice. Genes Dev. 2001; 15(4):467-81.

15. Zheng $\mathrm{Q}$, et al. Type $\mathrm{X}$ collagen gene regulation by Runx 2 contributes directly to its hypertrophic chondrocyte-specific expression in vivo. J Cell Biol. 2003;162(5):833-42.

16. Garcia AJ, et al. ERalpha signaling regulates MMP3 expression to induce FasL cleavage and osteoclast apoptosis. J Bone Miner Res. 2013;28(2):28390.

17. Nakatani T, Partridge NC. MEF2C interacts with c-FOS in PTH-stimulated Mmp13 gene expression in osteoblastic cells. Endocrinology. 2017;158(11):3778-91.

18. Shimizu E, et al. HDAC4 represses matrix metalloproteinase-13 transcription in osteoblastic cells, and parathyroid hormone controls this repression. J Biol Chem. 2010;285(13):9616-26.

19. Nishimura R, et al. Osterix regulates calcification and degradation of chondrogenic matrices through matrix metalloproteinase 13 (MMP13) expression in association with transcription factor Runx2 during endochondral ossification. J Biol Chem. 2012:287(40):33179-90.

20. Dreher $\mathrm{SI}$, et al. Significance of MEF2C and RUNX3 regulation for endochondral differentiation of human mesenchymal progenitor cells. Front Cell Dev Biol. 2020;8:81.

21. Komori T. Runx2, an inducer of osteoblast and chondrocyte differentiation. Histochem Cell Biol. 2018;149(4):313-23.

22. Kim EJ, et al. Ihh and Runx $2 /$ Run $\times 3$ signaling interact to coordinate early chondrogenesis: a mouse model. PLoS One. 2013;8(2):e55296.

23. Johnstone B, et al. In vitro chondrogenesis of bone marrow-derived mesenchymal progenitor cells. Exp Cell Res. 1998;238:265-72.

24. Meyer MB, et al. Epigenetic plasticity drives adipogenic and osteogenic differentiation of marrow-derived mesenchymal stem cells. J Biol Chem. 2016;291(34):17829-47.

25. Gebhard $\mathrm{S}$, et al. A highly conserved enhancer in mammalian type $\mathrm{X}$ collagen genes drives high levels of tissue-specific expression in hypertrophic cartilage in vitro and in vivo. Matrix Biol. 2004;23(5):30922.

26. Komori T. Regulation of skeletal development by the Runx family of transcription factors. J Cell Biochem. 2005;95(3):445-53.

27. $W u C$, et al. MicroRNAs play a role in chondrogenesis and osteoarthritis (review). Int J Mol Med. 2014;34(1):13-23. 
28. Zhang $Y$, et al. A program of microRNAs controls osteogenic lineage progression by targeting transcription factor Runx2. Proc Natl Acad Sci U S A. 2011;108(24):9863-8.

29. Gay I, et al. Differentiation of human dental stem cells reveals a role for microRNA-218. J Periodontal Res. 2014;49(1):110-20.

30. Chen S, et al. MicroRNA-218 promotes early chondrogenesis of mesenchymal stem cells and inhibits later chondrocyte maturation. BMC Biotechnol. 2019;19(1):6.

31. Zhao, C, Miao, Y, Cao, Z, et al. MicroRNA-29b regulates hypertrophy of murine mesenchymal stem cells induced toward chondrogenesis. J Cell Biochem. 2019:120:8742-53. https://doi.org/10.1002/jcb.28161.

32. Chen W, et al. "MicroRNA-381 Regulates Chondrocyte Hypertrophy by Inhibiting Histone Deacetylase 4 Expression." Int J Mol Sci. 2016;17(9):1377. https://doi.org/10.3390/ijms17091377.

33. Gabler J, et al. Stage-specific miRs in chondrocyte maturation: differentiation-dependent and hypertrophy-related miR clusters and the miR-181 family. Tissue Eng Part A. 2015;21(23-24):2840-51.

34. Bothe F, et al. Stimulation of calvarial bone healing with human bone marrow stromal cells versus inhibition with adipose-tissue stromal cells on nanostructured beta-TCP-collagen. Acta Biomater. 2018;76:135-45.

35. Benjamini $Y$, Hochberg $Y$. Controlling the false discovery rate: a practical and powerful approach to multiple testing. J R Stat Soc Ser B Methodol. 1995;57(1):289-300.

36. Dennis G Jr, et al. DAVID: database for annotation, visualization, and integrated discovery. Genome Biol. 2003;4(5):3.

37. Hecht $\mathrm{N}$, et al. Mechanosensitive MiRs regulated by anabolic and catabolic loading of human cartilage. Osteoarthr Cartil. 2019;27(8):1208-18.

38. Le Bleu HK, et al. Extraction of high-quality RNA from human articular cartilage. Anal Biochem. 2017:518:134-8.

39. Rao X, et al. An improvement of the $2^{\wedge}(-$ delta delta $C T)$ method for quantitative real-time polymerase chain reaction data analysis. Biostat Bioinforma Biomath. 2013;3(3):71-85.

40. Melnik S, et al. Cancer cell specific inhibition of Wnt/beta-catenin signaling by forced intracellular acidification. Cell Discov. 2018;4:37.

41. Winter $A$, et al. Cartilage-like gene expression in differentiated human stem cell spheroids: a comparison of bone marrow-derived and adipose tissuederived stromal cells. Arthritis Rheum. 2003:48(2):418-29.

42. Melnik S, et al. Impact of c-MYC expression on proliferation, differentiation, and risk of neoplastic transformation of human mesenchymal stromal cells. Stem Cell Res Ther. 2019;10(1):73.

43. Iwamoto $\mathrm{N}$, et al. Osteogenic differentiation of fibroblast-like synovial cells in rheumatoid arthritis is induced by microRNA-218 through a ROBO/Slit pathway. Arthritis Res Ther. 2018;20(1):189.

44. Gaur T, et al. Canonical WNT signaling promotes osteogenesis by directly stimulating Runx2 gene expression. J Biol Chem. 2005;280(39):33132-40.

45. Hassan $\mathrm{MQ}$, et al. miR-218 directs a Wnt signaling circuit to promote differentiation of osteoblasts and osteomimicry of metastatic cancer cells. J Biol Chem. 2012:287(50):42084-92.

46. Niehrs C. Function and biological roles of the Dickkopf family of Wnt modulators. Oncogene. 2006;25(57):7469-81.

47. Bovolenta $P$, et al. Beyond Wnt inhibition: new functions of secreted frizzledrelated proteins in development and disease. J Cell Sci. 2008;121(6):737-46.

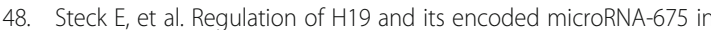
osteoarthritis and under anabolic and catabolic in vitro conditions. J Mol Med (Berl). 2012;90(10):1185-95.

49. Lu J, et al. MicroRNA-218-5p as a potential target for the treatment of human osteoarthritis. Mol Ther. 2017:25(12):2676-88.

50. Lu YF, et al. MiR-218 mediates tumorigenesis and metastasis: perspectives and implications. Exp Cell Res. 2015;334(1):173-82.

51. Reichenstein I, et al. Human genetics and neuropathology suggest a link between miR-218 and amyotrophic lateral sclerosis pathophysiology. Sci Transl Med. 2019;11(523):eaav5264.

52. Thiebes KP, et al. miR-218 is essential to establish motor neuron fate as a downstream effector of Is|1-Lhx3. Nature Communications. 2015;6(1):7718.

53. van der Kraan PM, van den Berg WB. Chondrocyte hypertrophy and osteoarthritis: role in initiation and progression of cartilage degeneration? Osteoarthr Cartil. 2012;20(3):223-32

\section{Publisher's Note}

Springer Nature remains neutral with regard to jurisdictional claims in published maps and institutional affiliations.

\section{Ready to submit your research? Choose BMC and benefit from:}

- fast, convenient online submission

- thorough peer review by experienced researchers in your field

- rapid publication on acceptance

- support for research data, including large and complex data types

- gold Open Access which fosters wider collaboration and increased citations

- maximum visibility for your research: over $100 \mathrm{M}$ website views per year

At BMC, research is always in progress.

Learn more biomedcentral.com/submissions 